\title{
FENÓMENOS DE TRANSFERENCIA ENTRE LENGUAS: EVIDENCIALIDAD EN EL ESPAÑOL EN CONTACTO CON EL GUARANÍ Y EL QUECHUA ${ }^{1}$
}

\author{
Alicia Avellana \\ Universidad de Buenos Aires, Comisión Nacional de Investigaciones Cienfíticas y Técnicas \\ alliciaavellana@yahoo.com.ar
}

Resumen

El presente trabajo describe, compara y analiza distintos valores evidenciales que adopta el pretérito pluscuamperfecto en dos variedades de contacto en la Argentina y Paraguay: el español en contacto con el que-chua y con el guaraní.

A partir de una descripción detallada de las gramáticas involucradas en el contacto determinamos, por un lado, si las construcciones consideradas se deben a procesos de transferencia. Por otro lado, especificamos el tipo de elemento gramatical transferido, dentro del marco teórico de la Gramática Generativa (Chomsky, 1981 y trabajos posteriores) y, especialmente, desde la propuesta de la Morfología Distribuida (Halle y Marantz, 1993, 1994, entre otros). Finalmente, evaluamos el alcance de este análisis para la sistematización de la variación lingüística dentro del modelo considerado.

PALABRAS CLAVE: contacto de lenguas; español-guaraní; español-quechua; evidencialidad; pretérito pluscuamperfecto.

\begin{abstract}
This paper describes, compares and analyses different evidential values conveyed by the Spanish pluperfect in two contact varieties in Argentina and Paraguay: Spanish in contact with Quechua and Guarani.

On basis on a detailed description of the grammars involved in each contact situation we examine, on the one hand, wether the constructions are due to tranference processes and, on the other hand, we determine the type of the grammatical element transferred, according to the framework of the Generative Grammar (Chomsky, 1981 and subsequent work) and, especially, the Distributed Morphology approach (Halle \& Marantz, 1993, 1994, among others). Finally, we evaluate the implications of this analysis for the sistematization of the interlinguistic variation within the generative framework.
\end{abstract}

KEY WORDS: language contact; SpanishGuarani; Spanish-Quechua; evidentiality; pluperfect.

\section{Introducción}

El presente trabajo describe, compara y analiza distintos valores modales que adopta el pretérito pluscuamperfecto en dos variedades del español en contacto con lenguas

\footnotetext{
${ }^{1}$ Quisierea agradecer la lectura y los valiosos comentarios de Laura Kornfeld.
} 
indígenas, dentro del marco teórico de la Gramática Generativa (Chomsky, 1981 y trabajos posteriores) y, en especial, desde la propuesta de la Morfología Distribuida (Halle y Marantz, 1993, 1994, entre otros).

Específicamente, trabajamos con datos del contacto con quechua en el noroeste de la Argentina y del contacto con guaraní en el nordeste de dicho país y en Paraguay. En particular, mostramos cómo una forma verbal similar en ambas variedades - y existente también en el español general- (i.e. el pretérito pluscuamperfecto) puede adoptar características sintáctico-semánticas y morfológicas propias de la lengua con la que está en contacto.

Así, a lo largo de este trabajo analizamos cómo el pretérito pluscuamperfecto manifiesta un valor evidencial, es decir, expresa distintos aspectos vinculados con la fuente mediante la cual se ha obtenido la información. Por un lado, en el contacto con el guaraní, codifica la sorpresa del hablante frente a un hecho no percibido previamente, como se ejemplifica en (1):

(1) a. Mirá vos, había sido que la pistola de Robocop sí existe [W1]. ('Resultó (ser) que la pistola de Robocop existe [pero yo no lo sabía hasta ahora].')

b. Se mudaron había sido... [W2] ('Resultó (ser) que se mudaron [pero yo no lo sabía hasta este momento].')

Por otro lado, en el contacto con el quechua adopta un tipo de valor evidencial diferente, que expresa que el hablante no ha experimentado personalmente los hechos que relata (2):

(2) a. Mi finado fue una vez a cazar a una laguna y ahí había estado ['estaba'], en un palo, la madre del agua, peinándose con un costillar de pescado. (...) Se había llegado ['llegó'] cerquita y vido que muy rubia había sido ['era']. [V:417, VIII] ('Mi finado fue una vez a cazar a una laguna y ahí estaba, en un palo, la madre del agua, peinándose con un costillar de pescado. (...) Se llegó cerquita y vio que era muy rubia [pero no tengo evidencia directa de ello].')

b. Le pegó otra vez. Lo agarra de la cabeza y va, lo mete en la pileta con agua. Lo baña bien.(...) Gritando había estado, el loro. [V:511, III] ('El loro estaba gritando [lo cual no me consta personalmente].')

A partir del análisis de datos como los de (1) y (2), en este trabajo nos proponemos, en primer lugar, determinar si puede hablarse efectivamente de transferencia por contacto, comparando en detalle las propiedades sintáctico-semánticas y morfológicas de estas estructuras con las de las lenguas indígenas correspondientes. En segundo lugar, examinamos el tipo de elementos que se traslada de una lengua a otra, con el objetivo de precisar qué aspectos gramaticales pueden resultar transferidos en el contacto. Finalmente, evaluamos el alcance que este análisis presenta para el estudio de la variación 
lingüística dentro del modelo generativo y, en especial, para la especificación de la noción de parámetro dentro de la propuesta de la Morfología Distribuida (Halle y Marantz, 1993, 1994, entre otros).

El trabajo se organiza de la siguiente manera: en el primer apartado, exponemos algunos aspectos vinculados con el marco teórico que resultarán útiles para el análisis posterior. El segundo y el tercer apartado están dedicados a la descripción y análisis de los datos del español en contacto con el guaraní y con el quechua respectivamente. En el cuarto apartado recapitulamos los distintos fenómenos de contacto y sistematizamos el tipo de modificación que producen sobre la gramática del español. Finalmente, en el último apartado, recogemos las conclusiones y examinamos sus consecuencias para la noción de variación dentro del marco teórico considerado.

\section{El marco teórico}

Este trabajo se inscribe dentro del marco teórico de la Gramática Generativa, un modelo formal en el que el estudio de la variación lingüística recibió un interés particular a partir de los años 80 con el modelo de Principios y Parámetros (Chomsky, 1981 y trabajos posteriores). Allí se propuso la noción de gramática universal para referirse a un componente gramatical innato conformado por un conjunto de principios universales, comunes a todas las lenguas. Estos principios, a su vez, se vinculan con una serie de parámetros, que son los que dan cuenta de la variación entre las lenguas dentro de los límites impuestos por la gramática universal.

La noción de parámetro, es decir, el elemento que permite formalizar la variación entre las lenguas, ha recibido distintas formulaciones desde su propuesta original. Así, en un comienzo se concebía como un conjunto de propiedades sintácticas abstractas que afectaba la aplicación de los principios universales en las lenguas particulares (como, por ejemplo, las propiedades vinculadas al parámetro del sujeto nulo en relación con el principio de proyección extendida). Sin embargo, en las propuestas más actuales los parámetros ya no se vinculan al componente sintáctico sino que afectan a las categorías funcionales presentes en cada lengua y, más específicamente, a los rasgos funcionales.

En esta investigación trabajamos en particular desde la propuesta de la Morfología Distribuida (Halle y Marantz, 1993, 1994, entre otros). Desde esta perspectiva, las gramáticas de las lenguas varían, en primer lugar, en cuanto a los rasgos que seleccionan dentro de un conjunto universal, que se denomina inventario universal de rasgos [Universal Feature Inventory]. Así, por ejemplo, existen lenguas que seleccionan un rasgo [dual], mientras que otras no lo hacen. En segundo lugar, las lenguas pueden variar en 
relación con distintas características que operan en el componente morfológico. Dicho componente - que constituye una de las propuestas innovadoras de la Morfología Distribuida - se ubica luego de la sintaxis y consiste en un conjunto de operaciones que son particulares a las lenguas (y que son puramente formales, es decir, que no reciben interpretación semántica). Por ejemplo, se aplica allí la operación de concordancia, que multiplica los mismos rasgos en distintas posiciones de la oración, o la operación de fusión, que une rasgos que luego serán expresados mediante un único morfema (como sucede en español, por ejemplo, con los rasgos de tiempo y aspecto en la morfología de pasado). Además de las operaciones mencionadas, en el componente morfológico se relacionan los rasgos abstractos con los exponentes fonológicos que los expresan en cada lengua (por ejemplo, se vincula el rasgo [plural] con sus realizaciones en español $s$, -es o $\varnothing$, o bien el rasgo [progresivo] con el exponente fonológico - $n d o$ correspondiente al gerundio). Los elementos que dan cuenta de la unión entre los rasgos abstractos y los exponentes fonológicos se conocen como ítemes de vocabulario ${ }^{2}$.

En suma, no se concibe un léxico inicial único que almacena toda la información paramétrica (como en los modelos generativos minimalistas) sino que esta se encuentra distribuida fundamentalmente entre la selección de rasgos del inventario universal y los distintos aspectos involucrados en el componente morfológico. A partir de esta propuesta teórica, en este trabajo indagamos sobre la posibilidad de que en el contacto de lenguas se transfieran distintas propiedades de la lengua fuente; en especial, discutimos si, a causa del contacto, puede modificarse, por un lado, la selección de rasgos sintáctico-semánticos y, por el otro, las distintas características morfológicas involucradas en el pretérito pluscuamperfecto. Específicamente, partimos de la hipótesis de que las transferencias pueden provocar transformaciones profundas en la gramática de una lengua. De este modo, defenderemos un análisis según el cual no solo las formas morfológicas superficiales pueden verse afectadas por el contacto sino también el conjunto de rasgos sintáctico-semánticos de una lengua.

\footnotetext{
${ }^{2}$ Técnicamente, los ítemes de vocabulario se formalizan de la siguiente manera: [progresivo] $\leftrightarrow /-$ ndo/. En este caso, el ítem ilustra para el gerundio la relación entre el rasgo [progresivo], que es resultado de las operaciones sintácticas y morfológicas previas, y el exponente fonológico -ndo que lo realiza en español.
} 


\section{El contacto con el guaraní}

Los datos con los que trabajamos en este apartado corresponden a hablantes bilingües español-guaraní y hablantes monolingües del español del nordeste de la Argentina (fundamentalmente de las provincias de Corrientes y Misiones) y de Paraguay ${ }^{3}$.

A continuación, nos abocamos al análisis del pretérito pluscuamperfecto de esta zona. En la primera sección describimos el fenómeno; en la segunda, contrastamos su funcionamiento con la construcción guaraní correspondiente. Finalmente, analizamos la forma verbal resultante del contacto en términos del marco teórico en el que se inscribe este trabajo.

\subsection{Descripción del fenómeno}

En toda el área en donde se habla guaraní se observa un uso muy productivo del pretérito pluscuamperfecto con valor modal ${ }^{4}$, bajo la forma había sido (que). El significado que expresa puede parafrasearse, aproximadamente, mediante la estructura resultó (ser) que del español general, y se encuentra en distintos tipos de hablantes y de registros en toda la zona del nordeste de la Argentina (3) y en Paraguay (4):

(3) a. Había sido que esa canción está cantado a un desaparecido5.

b. Mirá vos, había sido que la pistola de Robocop sí existe. [W1]

c. Había sido que Anabela Askar es de las bizarras cultas. [W3]

d. Había sido que me re copé escribiendo. [W4]

(4) a. Había sido que el pelado fue castigado. [W5]

b. Había sido que todavía no almorcé ...I love my job! [W6]

\footnotetext{
${ }^{3}$ A lo largo del trabajo incluimos, por un lado, datos propios, obtenidos en viajes de campo (a las provincias argentinas de Santiago del Estero y Corrientes y a Paraguay), mediante la observación participante y entrevistas semipautadas. Por otro lado, utilizamos también datos tomados de otros autores que han trabajado en la zona y de búsquedas en sitios web (cuyas referencias se indican entre corchetes al lado de cada ejemplo). Cuando no se indica la fuente, se trata de datos propios. La estructura que analizamos aquí está extendida en el español de esta área, no solo en hablantes bilingües sino también en monolingües de español, debido a que se trata de una zona de contacto prolongado con el guaraní en la que muchas transferencias se han integrado por completo en el español actual.

${ }^{4}$ Utilizamos en este trabajo el término modal en un sentido general que abarca distintos valores tales como la evidencialidad y la miratividad (como se usa, por ejemplo, en Cinque (1999)). Volveremos sobre estos conceptos con mayor detalle en $\$ 2.2$.

${ }^{5}$ Como se observa en este ejemplo, en esta variedad de contacto es frecuente la neutralización del género.
} 


\section{c. Había sido que Wang Chan Kein se murió ahorcado. [W7] \\ d. Pero no, ¡había sido que era ella!! [W8]}

Semánticamente, en (3) y (4) el pretérito pluscuamperfecto pierde el valor de anterioridad en el pasado que posee en el español general (cfr. Cuando yo llegué Mariana ya se había ido). Aquí, esta forma verbal expresa sorpresa frente un evento que sucedió y del cual el hablante no tuvo registro durante su ocurrencia sino que, por el contrario, lo percibe posteriormente. Así, (3a) debe interpretarse como 'Esa canción es sobre un desaparecido [pero yo no lo sabía hasta ahora]'. Este valor no se manifiesta en el español general de manera gramaticalizada sino léxicamente mediante estructuras como la ya mencionada resultó (ser) que (...) o al final (...), o bien con el uso del pretérito imperfecto acompañado por expresiones de asombro o de alguna entonación particular (Mirá vos/Ah, era sobre un desaparecido.).

En cuanto a su posición, la estructura había sido (que) se ubica al comienzo de la oración cuando lleva el complementante que (cfr. 3-4). Sin embargo, en el español de Paraguay ocurre más frecuentemente sin que, lo cual le otorga una mayor libertad posicional. En ese caso suele ubicarse al final de la oración, como se observa en (5), aunque puede ocurrir también al comienzo (6):

(5) a. Hay una máquina para estirar el alambre había sido.

b. Eras vos había sido.

c. Teníamos orden de captura había sido. [W9]

d. ¡No era simulación había sido!! [W10]

(6) a. Había sido tenía una novia bien guardadita. [W5]

b. Había sido todo sigue igual que cuando me fui. [W11]

c. ¡Había sido hay algunas linditas en el equipo! [W12]

d. Había sido era olimpero luego el tipo. [W13]

Asimismo, la estructura sin complementante puede posponerse a algún elemento dentro de la oración, como en (7), pudiendo incluso interrumpir la adyacencia entre el verbo y sus complementos (cfr. 7b):

(7) a. El trabajador del volante había sido estuvo laburando toda la noche y camino ya a su casa, le dio el patatús letal. [Pa:291]

b. Por errores del sistema no se pudo cargar había sido el número gratis. [W14]

c. Ganó un sueldazo había sido ahí.

d. A todos nos pasa había sido esto. [W14] 
En términos sintácticos, esta estructura modifica una oración completa, que puede llevar flexión de tiempo presente (8) o pasado (9), cualquiera sea la posición que ocupe en la oración:

(8) Presente

a. Había sido que de la costa oeste del Paraná también hay locos sueltos. [W1]

b. Había sido que los nuevos vecinos son coreanos. [GT]

c. Franko pio ${ }^{6}$ es holandés había sido. [W15]

(9) Pasado

Pretérito imperfecto

a. Había sido que era anoréxica.

b. Había sido que Guido Süller tenía sus novias. [W16]

c. Era su cumpleaños había sido. [An]

Pretérito perfecto simple

d. Hotmail no es "correo caliente". Había sido que Jack Smith eligió algunas letras de HTML [W17]

e. Había sido que se tomaron todo el vino.

f. Se mudaron había sido. [W2]

La diferencia entre el uso de la morfología de presente y de pasado, sin embargo, no siempre conlleva una distinción temporal. En particular, el pretérito imperfecto puede extenderse también hasta el presente (dado que no codifica perfectividad) y funcionar solo como un refuerzo del valor sorpresivo, como ocurre en el español general. Así, (9a) puede interpretarse con alcance sobre el momento de habla: 'Resultó ser que es anoréxica'. Esto contrasta con el pretérito perfecto simple, que codifica efectivamente un valor perfectivo, sin alcance sobre el presente.

Finalmente, otra característica de interés es que esta estructura funciona como una unidad indivisible y fosilizada: se construye siempre con el participio del verbo ser y no es productiva con otras clases léxicas ni flexiona en otros rasgos de número y persona.

Teniendo en cuenta lo anterior, los usos descriptos hasta aquí deben ser diferenciados de una estructura muy similar que se registra de manera extendida en el habla rural de varios países hispanoparlantes. Así, Kany (1969)7 documenta la estructura había sido,

\footnotetext{
${ }^{6}$ Variante de piko, préstamo del morfema guaraní que se utiliza en cierto tipo de interrogaciones.

${ }^{7}$ Kany (1969) propone que esta estructura podría deberse a una analogía con la forma ha de ser. Si ha de ser en muchas regiones significa 'será' (es decir, la forma presente de haber señala futuro) es posible que el imperfecto había se reinterprete como presente.
} 
con valores semánticos similares a los mencionados, tanto en la Argentina (10a) como en otros países (10b-f) y la atribuye al "habla gauchesca":

(10) a. ¡Cha que había sido salame! [Argentina] [Ka:206]

b. iPucha qui habían sido flojo los nacione! [Uruguay] [Ka:207]

c. Brava había sido Ud. ¿no? [Ecuador] [Ka:208]

d. Tu hijo había sido un mañudo. [Bolivia] [Ka:207]

e. ¡Qué mal pensado había sido usted, Don Juan Francisco! [Perú] [Ka:207]

f. Había sido una mujer. [Colombia] [Ka:208]

Las estructuras descriptas por Kany se encuentran también con cierta frecuencia en el español rural de la Argentina, incluso en zona de influencia guaraní:

(11) a. ¡Qué interesada había sido la virgen!! [V:186, VIII]

b. Encontró un guazuncho. Y habian sido dos. [V:760, IX]

c. Este viejito mendigo había sido Dios. [V:800, VII]

d. Ha visto que había sido mentira que era dura la cabeza de mi hermanito. $[\mathrm{V}: 643, \mathrm{VII}]$

Sin embargo, si comparamos en detalle la estructura descrita por Kany, que representamos en (12a), con la que analizamos aquí (12b) veremos que existen diferencias no triviales entre ambas; así, mientras que las estructuras del tipo de (12a) responden siempre al esquema había sido + sustantivo/adjetivo, las de (12b) poseen una forma invariable había sido (que) + oración:

(12) a. Había sido usted.

b. Había sido que era usted / Era usted había sido.

La diferencia esencial es que en (12a) el pretérito pluscuamperfecto constituye el verbo principal de la oración, de modo que concuerda con un sujeto referencial (cfr. Habían sido ustedes) mientras que en (12b), por el contrario, la forma invariable había sido (que) no es el verbo principal sino un modificador de la oración y, en consecuencia, no establece concordancia con el sujeto (cfr. Había sido que era usted / Había sido que eran ustedes).

El hecho de que la estructura había sido de (12b) posee un alcance oracional se ve reforzado por la posibilidad de que aparezca junto con que, imposible en (12a). Estas diferencias se explican a partir de una distinta estructura según la cual - como veremos en las próximas secciones- había sido (que) en (12b) no se comporta como un verbo pleno sino como un elemento funcional, una marca modal que gramaticaliza un rasgo evidencial. 
En suma, la expresión había sido (que) en la zona de contacto con el guaraní pierde el valor de anterioridad en el pasado ${ }^{8}$ adopta un significado modal. Asimismo, se ubica dentro de la oración en posiciones que resultan anómalas para el español general y, morfológicamente, se gramaticaliza de modo tal que deja de ser productivo junto a otras clases léxicas verbales y otros rasgos flexivos de número y persona.

En el próximo apartado analizaremos la relación de esta estructura con la gramática del guaraní para luego volver sobre su análisis teórico.

\subsection{Vinculación con el guaraní}

La estructura que hemos descripto en el apartado anterior es de frecuencia muy alta en toda el área de influencia del guaraní y parece, incluso, privativa de ella, al menos con las características que hemos delimitado.

A la frecuencia de aparición se suma la existencia de una construcción guaraní paralela a la descripta, lo cual favorece un análisis en términos de transferencia lingüística. En efecto, en guaraní existe un morfema ( $\left.r a^{\prime} e\right)$ que expresa un valor modal y normalmente junto con otros morfemas como niko/ningo o nipo/nimbo, que acompañan exclamaciones- adopta cierto matiz sorpresivo, como ilustran los siguientes ejemplos:

$\begin{array}{llllll}\text { (13) a. Lo } \quad \text { rico } & \text { r-a'y } & \text { nimbo } & \text { o-tĩ } & \text { avei } & \text { ra'e } \\ \text { Los rico } & \text { REL-hijo } & \text { NIPO } & \text { 3-avergonzarse } & \text { también } & \text { RA'E } \\ \text { 'Había sido que los hijos de los ricos también tienen vergüenza.' } & \\ \text { b. E'a, } & \text { ma'ẽ } & \text { Aniceto re-ju } & \text { nipo } & \text { ra'e } \\ \text { INTERJ } & \text { mirar } & \text { Aniceto 2sg-venir } & \text { NIPO } & \text { RA'E }^{\prime}\end{array}$

'Ea, mira Aniceto viniste pues (había sido)'. （Melià et al., 1997: 70)

${ }^{8}$ Conviene observar que, para indicar anterioridad en el pasado, en esta zona el pretérito pluscuamperfecto tiende a ser reemplazado por el pretérito perfecto simple (reforzado por la presencia del adverbio $y a$ ), como ocurre también en otras variedades:

(i) a. Eran tres hermanos; dos ya se fueron ['habían ido'] a rodar el mundo. [V:762, IX]

b. Y en ese fueron a visitarle a la madre que no le conoció porque creyó que ya murió ['había muerto'] con la torta envenenada. [V:764, IX]

c. Cuando vino la tormenta yo ya salí. ['había salido']. [GT]

${ }^{9}$ Las abreviaturas utilizadas en las glosas son las siguientes: 1, 2, 3: primera, segunda y tercera persona; BEN: benefactivo; CONT: continuativo; DIM: diminutivo; DUR: durativo; FUT: futuro; INTERJ: interjección; LIM: limitativo; LOC: locativo; NMZ: nominalizador; PAS.E: pasado experimentado; PAS.NE: pasado no experimentado; REL: relacionante; TOP: tópico. 
Según Krivoshein y Acosta (2007: 101), este morfema "indica el conocimiento que adquiere el hablante o su sorpresa al enterarse en un momento dado de la realización de una acción", es decir, se comporta de manera general como un morfema modal. Específicamente, asumiremos — siguiendo a Velázquez Castillo (2010) - que ra'e codifica un valor de evidencia inferida con extensiones mirativas, como precisaremos a continuación.

La evidencialidad es la categoría mediante la cual el hablante explicita la fuente de información (Aikhenvald, 2003), es decir, manifiesta si el hablante vio lo que relata (y, en consecuencia, tiene evidencia directa de ello) o si lo infirió o escuchó (y, en ese caso, posee evidencia indirecta). Existen lenguas para las cuales la naturaleza de la evidencia en la que se basa una afirmación debe ser especificada gramaticalmente en cada emisión mientras que otras, como el español, pueden expresarla solo léxicamente. Las lenguas varían en los sistemas de evidencialidad que presentan: algunas distinguen dos términos (visto $v s$. no visto o reportado $v s$. no reportado), mientras que otras pueden manifestar muchos valores más.

El morfema ra'e y, en especial, su correlato español habia sido (que) responde de manera ajustada a lo que se ha denominado "percepción demorada" [deferred realization] (Reuse, 2003). Este es un valor semántico de ciertos evidenciales que expresa que la información completa se obtuvo y se interpretó plenamente post-factum, sin que sea determinante el hecho de que el hablante haya efectivamente visto o no su ocurrencia (Aikhenvald, 2004). Por ejemplo, en (13b) el hablante posee evidencia directa para su afirmación; sin embargo, lo que está en juego es que dicha evidencia es utilizada para inferir un estado de cosas anterior no percibido en el momento de su ocurrencia.

En los ejemplos dados se observa, además, un valor de sorpresa sobre la percepción demorada, que indica que esta resulta inusual o inesperada para el hablante. Si se privilegia esta característica, ra'e cae también dentro de lo que se conoce como miratividad. Este valor ha sido recientemente descripto y codifica la "mente no preparada" [unprepared mind], es decir, lo que se percibe como información nueva no esperada y la sorpresa concomitante (Aikhenvald, 2004). La miratividad ha sido analizada como un valor diferenciado de la evidencialidad (cfr. DeLancey, 1997). Si bien la estructura había sido (que) podría ser considerada de este modo, creemos que la sorpresa se aplica aquí siempre sobre la percepción demorada y no de manera independiente. De este modo, una frase como Viniste había sido no manifiesta sorpresa solamente frente al hecho afirmado sino más bien frente a la percepción demorada del mismo. Aquí la miratividad (entendida en términos de codificación de la sorpresa) constituye un matiz semántico derivado, reforzado por la entonación y los elementos exclamativos, de la misma manera que en guaraní es la presencia del morfema exclamativo niko/nipo que aparece junto a 
ra'e lo que contribuye con dicho matiz (cfr. 13). Por todo esto, consideramos al rasgo codificado por el morfema ra'e un evidencial de "percepción demorada" con extensiones mirativas.

Por otra parte, si bien algunos autores (Liuzzi y Kirtchuk, 2010; Dietrich, 2010, entre otros) clasifican al morfema ra'e como una marca de pasado, siguiendo a VelázquezCastillo (2010) asumimos que la interpretación temporal surge solo como una extensión natural de su valor modal. De hecho, este puede referirse al presente (cuando el hecho se extiende hasta el momento de su percepción), como en (13a), o aparecer en un contexto de futuro como en (14), por lo que el tiempo deíctico no parece una limitación para su aparición:

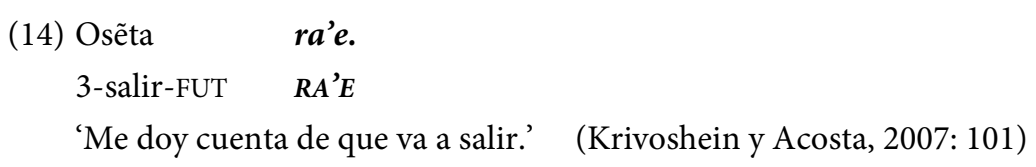

A partir de esto, la equivalencia entre ra'e y había sido (que) resulta evidente. Así, Palacios Alcaine (2008:291) afirma que "en la variedad coloquial paraguaya ra'e se traduce por había sido, forma que en esta variedad acentúa el carácter sorpresivo del hablante al transmitir una información que desconocía". Esto es consistente con la sistematicidad con la que los hablantes e investigadores de guaraní traducen una forma por otra (cfr. 13).

De modo similar, en el español de Paraguay se incorpora el morfema $r a^{\prime} e$ como préstamo en construcciones equivalentes a las que hemos ejemplificado con había sido $(q u e)^{10}$ :

(15)a. jLa nueva Miss Mundo quería ser monja ra'e! [W18]

b. Winnie the Pooh es ladrón ra'e. [W5]

c. 28 años es la edad perfecta de la mujer ra'e. [W5]

d. Su amada esposa ra'e tenía otro marido. [W5]

Asimismo, es posible encontrar ambas formas ( $r a$ 'e/había sido (que)) en el español de Paraguay en coexistencia dentro de la misma oración, de manera adyacente (16a-b) o no (16c-d), para expresar el mismo valor. Esta duplicación de las estructuras que son paralelas en las dos lenguas es muy frecuente en las situaciones de contacto:

${ }^{10}$ De manera inversa, lo mismo sucede en guaraní, que incopora como préstamo la forma del español: (i) Había sido la che dúo iñakã raku peteĩ morocha porãre oikóvo ra'e (Había sido que mi amigo se enamoró perdidamente de una bella morena) [W59] (La traducción corresponde al mismo hablante.). 
(16)a. El origen de las hortalizas era rapailandia, había sido ra'e.[W20]

b. Y había sido ra'e cuando hacés dieta tu cuerpo debe acostumbrarse a ella para abandonarla o, si no, subís el doble. [W5]

c. Había sido que a un ganadero francés lo "suicidaron" ra'e... [W5]

d. Había sido Aquiles era el problema ra'e. [W15]

En consecuencia, la similitud entre ra'e y había sido (que) ocurre, por un lado, en el nivel sintáctico-semántico, dado que ambos codifican percepción demorada con un matiz mirativo según el cual el hablante expresa sorpresa frente a un hecho no percibido previamente. Por otro lado, la forma había sido (que) funciona como un único morfema gramaticalizado, que modifica a la oración en su conjunto, tal como ocurre con ra'e. Asimismo, estas formas se asemejan en cuanto a la posición que ocupan: la estructura española calca normalmente la posición final (o pospuesta a algún constituyente) del morfema $r a^{\prime} e$, lo cual resulta inusual para el español general. De este modo, si bien las estructuras son superficialmente muy distintas en cada una de las lenguas (un morfema libre en guaraní frente a una perífrasis verbal en español), la equivalencia semántica que se establece entre estas conlleva también transferencias de tipo más superficial.

En suma, a pesar de que algunos autores como Granda (1988) -basándose en la existencia de las estructuras descritas por Kany (1969) (cfr. 10-11) - proponen que no es correcto postular una relación con el guaraní para este fenómeno, creemos que hay suficiente evidencia como para considerarlo un proceso de transferencia. La construcción que analizamos aquí se ha visto sin dudas favorecida y gramaticalizada por la presencia del guaraní y ha adoptado en esta área de influencia características particulares que la diferencian de los usos existentes en otras variedades.

En el próximo apartado nos detendremos en el análisis teórico de la estructura sintáctica y de los aspectos morfológicos vinculados con esta construcción.

\subsection{Estructura sintáctica y morfológica de la construcción de contacto}

Teniendo en cuenta la descripción provista en los apartados anteriores, adoptamos un análisis según el cual la expresión había sido (que) se ha gramaticalizado en esta zona; en particular, ha dejado de funcionar como un núcleo verbal para convertirse en un elemento funcional con valor modal. Esta construcción presenta diferencias respecto del pretérito pluscuamperfecto del español general, que pueden ubicarse tanto en el nivel de los rasgos sintáctico-semánticos como en la morfología que los realiza, como detallaremos a continuación. 
En el nivel abstracto, la construcción introduce un nuevo rasgo en el español, que denominaremos [percepción demorada]. De esta manera, esta variedad de español amplía su conjunto de rasgos activos a causa del contacto con la lengua indígena.

El rasgo mencionado se incorpora en la sintaxis sobre una proyección modal. En particular, desde la propuesta de Cinque (1999) — quien identifica las distintas proyecciones que se ubican por encima del Sintagma de Tiempo- este corresponde a una de las categorías más altas, es decir, aquellas que se refieren a la opinión o actitud del hablante respecto de la proposición:

(17) Modo (acto de habla)(M1) $>$ Modo (evaluativo)(M2) $>$ Modo $_{(\text {evidencial)(M3) }}>$ Modo (epistémico)(M4)

Específicamente, el rasgo [percepción demorada] debería vincularse con la proyección de Modo (evidencial), que indica el tipo de evidencia que tiene el hablante sobre lo expresado en la proposición. En otras palabras, proponemos que la expresión había sido (que) codifica un rasgo [percepción demorada] que se aloja en el núcleo de una proyección modal evidencial. De esta manera, esta estructura no constituye una proyección verbal sino un núcleo funcional más alto, con alcance sobre el resto de la oración. De esto se desprende, en consecuencia, que todos los ejemplos que hemos presentado en los apartados anteriores deben ser analizados como oraciones simples, con un único verbo pleno y en los que la estructura había sido (que) es una marca modal en el dominio de esa proyección verbal. La gran movilidad que presenta esta forma en la variedad de contacto es la habitual en las expresiones de este tipo, que se ubican en las proyecciones más altas de la oración.

En el nivel morfológico, por su parte, la incorporación del rasgo [percepción demorada] provoca una reorganización de los ítemes de vocabulario del español general (cfr. $\$ 1$ ). Para su expresión, como hemos observado, se toma en ocasiones el morfema ra'e como préstamo de la lengua indígena (cfr. 16). Sin embargo, lo más frecuente es que se resignifique una forma ya existente en español como lo es el pretérito pluscuamperfecto. Así, la estructura había sido (que) sufre un proceso de gramaticalización y se fosiliza como un único exponente fonológico, ligado a un nuevo rasgo. En otras palabras, la variedad de español en contacto con el guaraní incorpora un nuevo ítem de vocabulario, inexistente en el español general, que vincula el rasgo [percepción demorada] con el exponente fonológico había sido (que). En este sentido, el pretérito pluscuamperfecto del español general solo se asemeja superficialmente a la estructura que analizamos aquí, dado que codifica rasgos que había sido (que) no manifiesta (como, por ejemplo, rasgos aspectuales y de anterioridad). 
Es interesante notar que la equivalencia entre el guaraní y el español de contacto se establece en el nivel sintáctico-semántico y no en el de los exponentes fonológicos, que resultan muy distintos en cada lengua: en español general se trata de una forma perifrástica, compuesta por un conjunto de morfemas frente a un único morfema libre en guaraní. Las diferencias superficiales no impiden, sin embargo, la transferencia de rasgos, como observa Sánchez (2004) en el español en contacto con el quechua. Por otra parte, una vez seleccionada la expresión fonológica del español que expresará el nuevo rasgo $^{11}$, esta puede calcar ciertas características de la lengua indígena como, por ejemplo, la posición pospuesta.

En suma, el análisis de la estructura había sido (que) muestra que la gramática del español en contacto con el guaraní se ha modificado en los dos niveles analizados. Por un lado, en el nivel de los rasgos abstractos, ha ampliado su inventario al incorporar un nuevo rasgo: [percepción demorada]. Esto confirma —en contra de lo que sostienen muchos autores- que el contacto de lenguas no provoca solamente simplificaciones sobre las gramáticas de contacto sino que los sistemas pueden verse enriquecidos con rasgos gramaticales innovadores. Asimismo, apoya el hecho de que las transferencias entre lenguas no son meramente superficiales sino que pueden producir cambios gramaticales profundos en las variedades de contacto. Por otro lado, en el nivel de la morfología superficial, hemos verificado también ciertas transferencias, que provocan reestructuraciones sobre el conjunto de los ítemes de vocabulario del español. A partir de esto, podemos concluir que la estructura había sido (que) ha adoptado características peculiares en ambos niveles analizados, que son resultado de la presencia de la lengua indígena y de su interacción con las propiedades del español, generándose así una nueva gramática de contacto.

\footnotetext{
${ }^{11}$ Es posible que la elección de la forma del pret. pluscuamperfecto para dar cuenta de este nuevo rasgo no sea totalmente azarosa. Este tiempo verbal en español indica anterioridad en relación con un momento de referencia, de modo similar a ra'e, que manifiesta la percepción posterior de algo normalmente ya sucedido. De todos modos, no es la mayor similitud lo determinante en dicha elección. Así, no se explica por qué se prefiere la gramaticalización de dicha forma verbal frente a, por ejemplo, el uso del pretérito imperfecto, que ya expresa valores similares en el español general (acompañado de otros elementos que refuerzan dichos valores pragmáticos; cfr. jAh, eras vos!).
} 


\section{El contacto con el quechua}

En cuanto a la situación de contacto con el quechua, trabajamos con datos de hablantes bilingües español-quechua y monolingües de español correspondientes al noroeste de la Argentina (principalmente a la provincia de Santiago del Estero) ${ }^{12}$.

\subsection{Descripción del fenómeno}

Herminia Martín (1976) inicia su artículo "Un caso de interferencia en el español paceño" con el siguiente ejemplo, que ilustra en el español boliviano ${ }^{13}$ el uso del pretérito pluscuamperfecto al que nos dedicaremos en este apartado:

(18) A: - Sapir fue un gran lingüista.

B: - ¿Cómo lo sabes?

A: - Los libros lo dicen.

B: - Entonces no puedes hablar así; debes decir: Sapir había sido un gran lingüista.

(Herminia Martín, 1976: 119)

En la Argentina, el valor del pretérito pluscuamperfecto ejemplificado en (18) aparece fundamentalmente en zonas rurales que están y/o han estado en contacto con el quechua. Mediante esta forma verbal se indica que el hablante no presenció los hechos que relata, como se ejemplifica en las siguientes oraciones correspondientes al español rural de Santiago del Estero y de otras provincias del noroeste argentino:

(19) a. Se había llegado ['llegó'] cerquita y vido que muy rubia había sido ['era']. [V:417, VIII]

b. Gritando había estado ['estaba'], el loro. [V:511, III]

c. $Y$ entonces advierte él que andaba arriba un cuervo y dice:

- ¿Sabe que me voy a hacer el muerto?

\footnotetext{
${ }^{12}$ Cabe remarcar que, si bien hay hablantes bilingües en esta zona, la estructura que analizamos se debe fundamentalmente a una situación de sustrato; en otras palabras, la presencia del quechua a lo largo de los siglos ha dejado huellas sobre el español de la zona que son prácticamente independientes de su vitalidad actual. No obstante, estos usos se reservan, en el español de la Argentina, a contextos rurales y no poseen un uso extendido en la variedad regional, a diferencia de lo señalado para el contacto con el guaraní.

${ }^{13}$ Este ejemplo corresponde al contacto con el aymara. No obstante, el fenómeno que analizamos aquí es idéntico a lo que ocurre en esta lengua indígena ( $y$ en el español en contacto con ella).
} 
Y así había hecho ['hizo']. Se había tirado ['tiró'] en el campito y había dado ['dio'] vuelta al sieso. [V:391, III]

d. Y por ahí había encontrado ['encontró'] una higuera. [V:26, VI]

Los verbos remarcados en (19) corresponden a formas de pasado simples en español general, como se indica entre corchetes. Semánticamente, el pretérito pluscuamperfecto pierde aquí también el valor de anterioridad en el pasado para utilizarse como un pasado narrativo no experimentado, es decir, una forma que agrupa pasado y evidenciali$\operatorname{dad}^{14}$. De esta manera, aunque abandona el rasgo temporal anafórico, mantiene un rasgo de tiempo deíctico, que codifica pasado respecto del momento de la enunciación.

Específicamente, este tiempo verbal expresa evidencia indirecta, es decir, manifiesta que el hablante no ha experimentado los hechos que relata. Así, por ejemplo, (19a) debe interpretarse como 'Llegó cerquita y vio que era muy rubia [pero no tengo evidencia directa de ello]' o (19b) como 'El loro estaba gritando [lo cual no me consta personalmente]'.

Dado que se trata aquí de un pasado narrativo, su uso no se reduce a oraciones aisladas en contextos discursivos acotados (como sucede con había sido (que) en el contacto con el guaraní, que se utiliza en contextos exclamativos) sino que aparece de manera reiterada a lo largo de una narración. Asimismo, el pretérito pluscuamperfecto se utiliza en esta zona de manera productiva con distintas raíces verbales y no se limita al verbo ser. Estas dos características aparecen ejemplificadas en el siguiente fragmento narrativo. Allí se observa la repetición de las formas del pluscuamperfecto utilizado como tiempo de la narración y su productividad con distintas raíces verbales:

(20) Mi finado fue una vez a cazar a una laguna y ahí había estado, en un palo, la madre del agua, peinándose con un costillar de pescado. Y ahí la ha hallado. Se había llegado cerquita y vido que muy rubia había sido. Dice que había tenido la cara delgadita, como una criatura chica y muy bonita había sido. Pero las trenzas decía que las tenía, po, muy largas, que habían estado topando el agua, y ella había tendido casi todo el cuerpo afuera, y desnudo que había estado. Y decía que después de un rato que se había volcau al agua, y que el agua había quedado batiéndose. [V:417, VIII]

\footnotetext{
${ }^{14}$ En otras variedades del español andino algunos autores señalan que es el pretérito perfecto compuesto el que manifiesta valores evidenciales. Según Kany (1969) este tiempo verbal funciona en Ecuador como un evidencial de presente y el pretérito pluscuamperfecto como un evidencial de pasado: Bastantes muebles ha tenido ['tiene'] / Habían sido ['eran'] los hijos de unos señores de las haciendas de Machachi [Kany, 1969:208].
} 
Asimismo, se desprende de los ejemplos que los verbos flexionados en pretérito pluscuamperfecto se comportan aquí como verbos plenos, es decir, son los elementos que determinan la estructura argumental de cada oración y portan los morfemas de concordancia con el sujeto.

Otra de las características que puede resaltarse del pretérito pluscuamperfecto en el contacto con el quechua es que bajo esta forma se neutraliza la oposición aspectual perfectivo/imperfectivo propia del tiempo pasado en el español general. En efecto, esta construcción verbal cubre los valores correspondientes tanto al aspecto perfectivo (21) como al aspecto imperfectivo (22), como se observa en los siguientes ejemplos:

(21) a. Y por ahí había encontrado ['encontró'] una higuera. [V:26, VI]

b. Y diz que el tigre nada había dicho ['dijo'] [V:278, I]

c. Habían salido ['salieron'] corriendo carrera. [V:418, II]

d. Se había llegado ['llegó'] cerquita (...) [V:417, VIII]

(22) a. Gritando había estado ['estaba'] el loro. [V:511, III]

b. Había habido ['había'] una laguna cerca. [V:461, II]

c. Y por ahí habían estado ['estaban'] cantando las chuñas. [V:608, III]

d. Había estado ['estaba'] Pedro, embolsado sobre un río. [V:204, VIII]

Asimismo, como se observa en (21b), estos usos evidenciales del pretérito pluscuamperfecto coexisten frecuentemente con las formas diz que/dice que/que, que especifican que la información que tiene el hablante proviene de algo que este ha oído, como en los siguientes ejemplos:

(23) a. Diz que habían sido ['eran'] dos hermanos. [V:679, VII]

b. Que era como pa partirla con la uña, diz que había sido ['era']. [V:278, I]

Por otra parte, esta forma verbal puede adoptar un valor mirativo en algunos contextos ${ }^{15}$, en los que se utiliza también de manera productiva con distintas raíces verbales $(24 \mathrm{a}-\mathrm{c})$, incluyendo la cópula ser (24d-f):

(24) a. ¡Qué muchos niñitos y tan lindos que había tenido, señora! [V:213, I]

b. Los difuntos habían estado muy caros. [V:223, VIII]

c. Lo había metido mal. [V:293, VIII]

d. ¡Bah! No había sido médico. [V:547, III]

\footnotetext{
${ }^{15}$ En el español de Ecuador, en donde el pretérito perfecto compuesto puede adoptar un valor evidencial, este puede codificar también un valor mirativo: Mire, compró estos, los probé y ¡han sido peras! (Olbertz, 2009: 70).
} 
e. El hueso había sido de león. [V:430, VII]

f. Había sido la casa de los ladrones. [V:44, VIII]

Cuando expresa un valor mirativo como en (24), el pretérito pluscuamperfecto puede extenderse hasta el presente, al igual que sucede en el contacto con el guaraní. Así, (24a) debe entenderse como ‘¿Cuántos y qué lindos niñitos que tiene, señora!', con alcance sobre el momento de enunciación.

La utilización del pretérito pluscuamperfecto como pasado no experimentado conlleva, a su vez, una reorganización de todo el sistema verbal. Así, este tiempo se opone al del pretérito perfecto compuesto ${ }^{16}$, que se vuelve la forma no marcada respecto de la evidencialidad.

Finalmente, de manera similar a lo que sucede en el contacto con el guaraní (cfr. nota al pie (7)), el pretérito pluscuamperfecto tiende a ser reemplazado por el pretérito perfecto simple/compuesto - acompañado de la forma ya- para expresar anterioridad en el pasado:

(25)a. Y, claro, ya si ha dado ['había dado'] cuenta de las intenciones del compadre. $[\mathrm{V}: 212, \mathrm{I}]$

b. Pero ha pasado de alto una parte, ella ya lo alcanzó a ver ['había alcanzado a ver'] también. [V:390, VIII]

c. Y ya se dio cuenta ['había dado cuenta'] que venía el sapo. [V:497, II]

Por lo demás, el pretérito pluscuamperfecto con valor evidencial no presenta más restricciones relevantes que las observadas. Lo descrito hasta aquí, sin embargo, resulta suficiente para distinguir las estructuras del contacto con el quechua de las resultantes del contacto con el guaraní, por un lado, y de los "usos gauchescos" presentes de manera más extendida en otras regiones de la Argentina (cfr. 10-11), por el otro, como argumentaremos a continuación.

Una de las diferencias esenciales entre la estructura de contacto con el quechua y la de contacto con el guaraní se da en el nivel sintáctico-semántico. El valor evidencial que

\footnotetext{
${ }^{16}$ En esta zona existe una tendencia a neutralizar (aunque no de modo absoluto) la oposición entre el pretérito perfecto simple y el compuesto en favor de este último, de modo exactamente opuesto a lo que ocurre en la mayor parte de la Argentina. Así, el pretérito perfecto compuesto se convierte en la forma no marcada del pasado y puede, en consecuencia, señalar hechos ya concluidos, sean estos cercanos o no al momento de habla:

(i) Y ha seguido el tigre persiguiéndolo al zorro. Y se le disparaba siempre, hasta que ha resuelto hacerse el muerto. Y se ha hecho el muerto. Y la tigra ha llamado a todos los animales. Y lo han llamado al zorro (...). [V:292, I]
} 
codifica cada una de estas estructuras es distinto y esto explica las asimetrías en los contextos discursivos en los que aparece cada una: mientras que en el guaraní su aparición está acotada a contextos exclamativos, que indican sopresa, en el contacto con el quechua se reitera a lo largo de una secuencia narrativa. Asimismo, otra diferencia en este nivel es que, si bien en ambas situaciones el pretérito pluscuamperfecto pierde su valor de anterioridad en el pasado, en el contacto con el quechua mantiene un rasgo temporal de pasado deíctico.

Por otra parte, en relación con las propiedades morfológicas, en el contacto con el guaraní constituye una estructura fosilizada que se expresa de manera separada del verbo principal, es decir, funciona como un morfema aislado, portador de un único rasgo modal. En el quechua, por el contrario, el rasgo evidencial se fusiona con la morfología temporal y constituye el verbo principal de la oración, aquel que aporta la estructura argumental. En consecuencia, no se limita a la construcción con el verbo ser sino que es productivo con distintas raíces verbales. Estas diferencias repercuten, a su vez, en la posición que ocupa la estructura en cada caso. En el contacto con el guaraní, al tratarse de un morfema libre, correspondiente a un valor modal, posee una libertad mucho mayor, que calca usualmente la posición pospuesta del morfema guaraní. En el contacto con el quechua, por el contrario, presenta las restricciones posicionales propias del verbo principal de la oración.

En cuanto a los "usos gauchescos" (cfr. 10-11), por su parte, encontramos dos diferencias sustanciales: en relación con los rasgos sintáctico-semánticos, los usos descritos por Kany (1969) se limitan a valores mirativos ${ }^{17}$, mientras que los que se hallan en zona de contacto con el quechua funcionan como un pasado narrativo; en segundo lugar y en relación con lo anterior, en el contacto con el quechua la forma es productiva con distintas raíces verbales y no se limita a la construcción con ser como sucede con los otros.

En el próximo apartado examinamos el valor que posee la estructura correspondiente en quechua y establecemos las similitudes y diferencias existentes con el español, con el objetivo de determinar su posible vinculación.

\subsection{Vinculación con el quechua}

La estructura que hemos descrito en el apartado anterior se registra en toda el área del español andino, tanto con su valor de pasado no experimentado como cuando pre-

\footnotetext{
${ }^{17}$ Olbertz (2005) señala algo similar para el español de Ecuador, en donde solo los usos mirativos puros se han extendido al español estándar regional.
} 
senta extensiones mirativas. En la bibliografía, numerosos autores (Schumacher de Peña, 1975; Hardman de Bautista, 1982; Granda, 1988; Klee y Ocampo, 1995; Cerrón Palomino, 2003; Sánchez, 2004; Merma Molina, 2007, entre muchos otros) vinculan estos usos del pretérito pluscuamperfecto con el morfema evidencial quechua -sqa (o, en algunas zonas, con el morfema paralelo en aymara).

En líneas generales, el morfema -sqa manifiesta que el hablante no posee evidencia directa para aquello que afirma (26), en oposición a su contraparte -rqa (27):

(26) Para-sha-sqa

llover-DUR-PAS.NE

'Estaba lloviendo.' (el hablante escuchó/infirió que estaba lloviendo.)

(27) Para-sha-rqa-n

llover-DUR-PAS.E-3

'Estuvo lloviendo.' (el hablante vio que estuvo lloviendo.) (Faller, 2004: 46)

En efecto, en (26) -sqa expresa que el hablante no posee evidencia directa de que ha llovido sino que conoce esta información porque se la han dicho o porque la ha inferido a partir del resultado de la lluvia; por el contrario, en (27) -rqa señala que el hablante posee evidencia directa para su afirmación. De este modo, asumimos aquí que -sqa codifica un "pasado no experimentado", es decir, manifiesta que el hablante no ha experimentado personalmente los hechos que relata, sin especificar si estos han sido reportados o inferidos ${ }^{18}$.

En relación con lo anterior, en quechua es posible especificar que el hablante ha obtenido la información a partir del relato de otros hablantes. Para esto, se utiliza el enclítico reportativo si/s, que indica que el hablante adquirió la información porque la escuchó. Las formas -sqa y si coexisten con frecuencia, como se observa en el siguiente ejemplo:

(28) wawa-cha

ka-sha-qti-lla-y-raq-si

tiyu-y-qa

bebé-DIM

ser-DUR-NMZ-LIM-1-CONT-REP

tío-1-TOP

\footnotetext{
${ }^{18}$ No todos los autores analizan este morfema de igual modo. Así, este ha recibido distintas denominaciones que privilegian algún matiz de significado, entre las que se encuentran "pasado reportativo" (Cusihuamán, 1976), y “tiempo de descubrimiento repentino" [sudden discovery tense] (Adelaar, 1977; Lefebvre y Muysken, 1988), además de "pasado no experimentado" (Cerrón Palomino, 1994), que adoptamos aquí. Por su parte, Faller (2004) propone que se trata de un elemento deíctico, que ubica la situación fuera del campo perceptual del hablante; en consecuencia, el valor evidencial que posee es, para la autora, solo una interpretación derivada del valor espacial.
} 
wañu-pu-sqa

morir-BEN-PAS.NE

'Mi tío murió cuando yo era todavía un bebé'.

(Faller, 2004: 52)

El valor reportativo de si exige, cuando el relato está en pasado, el morfema -sqa. Este reportativo, como indican Albarracín et al. (2002: 152) se traduce normalmente en español por dicen que/diz que/se dice que.

Por otra parte, el morfema -sqa puede aportar también un valor mirativo, lo cual ha dado lugar al término "pasado de descubrimiento repentino" [sudden discovery tense] (Adelaar, 1977; Lefebvre y Muysken, 1988) (cfr. nota 17), como en los siguientes ejemplos:

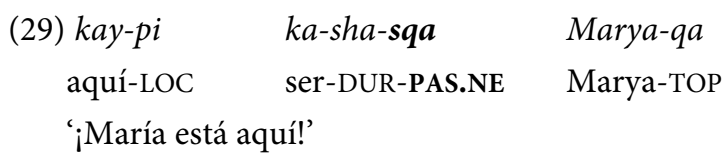

(Faller, 2004: 53)

De esta manera, como un pasado narrativo puro, el morfema -sqa señala una acción que el hablante no ha experimentado de manera directa o bien sobre la cual no ha tenido control (durante un sueño, por ejemplo); cuando posee una extensión mirativa, expresa sorpresa sobre la percepción del hecho que se relata. En este último caso, el hablante puede hacer referencia a una situación que se extiende hasta el presente.

Por último, -sqa fusiona los valores de pasado y evidencialidad en un único afijo que, morfológicamente, se sufija a cualquier base verbal, como se observa en los siguientes ejemplos:

(30)a. Tapusqa ['él había preguntado'].

b. Nipusqa ['él le había dicho'].

c. Sorqosqa ['él había sacado'].

d. Tarisqa ['él había encontrado’]. (Albarracín et al., 2002: 106)

De lo expuesto hasta aquí se desprende un comportamiento paralelo entre el morfema -sqa y el pretérito pluscuamperfecto del español de contacto: por un lado, se asemejan semánticamente, en tanto ambas formas codifican un pasado deíctico con evidencia indirecta que puede, en algunos contextos, poseer extensiones mirativas. Por otro lado, este morfema en quechua se aplica de manera productiva sobre distintas raíces verbales, al igual que sucede con el tiempo del español, para conformar el verbo principal de la oración. Asimismo, el morfema quechua aparece en el contexto del clítico reportativo $s i$, tal como ocurre con las distintas formas de decir en la variedad que analizamos. De modo paralelo al comportamiento de los morfemas -sqa y si en quechua, la diferencia fundamental entre el uso del pretérito pluscuamperfecto y el del 
verbo decir reside en que el primero codifica que la información no le consta al hablante, mientras que el segundo explicita que el conocimiento ha sido adquirido de oídas. De esta manera, siguiendo a Hardman de Bautista (1982), las diferencias modales que pueden establecerse en las variedades andinas como la que analizamos aquí son las siguientes:

(31)a. Ella ha comprado la casa

b. Ella había comprado la casa

c. Ella había comprado la casa dice
'Ella compró la casa [lo cual me consta].'

'Ella compró la casa [lo cual no me consta].'

(Adaptado de Hardman de Bautista, 1982)

De esto se desprende que el uso del pretérito pluscuamperfecto para señalar evidencia indirecta supone una oposición modal respecto del pretérito perfecto compuesto. Así, no es solo la incorporación de un nuevo rasgo lo que está en juego aquí sino la gramaticalización de todo un sistema evidencial que establece nuevas oposiciones entre las formas ya existentes.

En el próximo apartado precisaremos en términos teóricos cuál es la estructura gramatical resultante del contacto.

\subsection{Estructura sintáctica y morfológica de la construcción de contacto}

En este caso, al igual que en el contacto con el guaraní, el quechua provoca modificaciones tanto en el nivel de los rasgos sintáctico-semánticos como en el de la morfología del español.

Por un lado, en cuanto a la selección de rasgos abstractos, el pretérito pluscuamperfecto codifica en esta zona un nuevo rasgo, que denominaremos [evidencia indirecta], que expresa que el hablante no experimentó la situación que relata. De este modo, la presencia de la lengua indígena genera una ampliación de la lista de rasgos activos en esta variedad del español. Este nuevo rasgo se incorpora en la sintaxis sobre un sintagma modal, que siguiendo la propuesta de Cinque (1999), correspondería también a la proyección de Modo (evidencial) ${ }^{19}$ (cfr. 17).

Por otra parte, además de sumar un valor modal, hemos observado que este uso del pretérito pluscuamperfecto presenta diferencias temporales respecto del español gene-

\footnotetext{
${ }^{19}$ En cuanto a los valores mirativos, asumiremos aquí (siguiendo a Faller, 2007) que estos son una extensión pragmática del valor evidencial inherente de -sqa, de modo similar a lo que ocurre con este significado en el pretérito imperfecto del español general.
} 
ral, dado que no codifica un tiempo anafórico de anterioridad sino solamente un pasado deíctico. Así, en español general, se propone que este tiempo verbal implica dos proyecciones temporales: la correspondiente al tiempo deíctico, o $\mathrm{T}_{1}$ (que aloja el rasgo [pasado]) y la correspondiente a $\mathrm{T}_{2}$, que codifica el tiempo anafórico (con el rasgo [anterior]) (cfr. Giorgi y Pianesi, 1997; Kornfeld, 2004). En el contacto con el quechua, por el contrario, no se proyectan dos nodos temporales sino que se despliega únicamente el nodo deíctico $\left(\mathrm{T}_{1}\right)$ (con el rasgo [pasado]), sumado al nodo evidencial (con el rasgo [evidencia indirecta]).

Asimismo, la sintaxis del pretérito pluscuamperfecto en el español de contacto es neutral en cuanto a la oposición perfectivo/imperfectivo dado que, como hemos observado, puede ser equivalente tanto al pretérito perfecto simple como al pretérito imperfecto del español general (cfr. 21-22).

En suma, el contacto provoca las siguientes modificaciones en el dominio sintáctico de esta variedad ${ }^{20}$ : (i) introducción de una proyección de Modo (evidencial), que aloja el rasgo [evidencia indirecta]; (ii) presencia de un único nodo temporal $\left(\mathrm{T}_{1}\right)$, que da cuenta del rasgo deíctico [pasado]; (iii) ausencia de especificación aspectual.

A su vez, estas modificaciones sobre el conjunto de rasgos sintáctico-semánticos provocan reorganizaciones en los elementos morfológicos superficiales que los expresan. Los nuevos rasgos no se manifiestan mediante un préstamo de la lengua indígena - como ocurre, por ejemplo, en el contacto con el guaraní con el morfema ra'e- sino únicamente mediante la resignificación de los ítemes de vocabulario correspondientes a las formas del pretérito pluscuamperfecto ${ }^{21}$. Así, mientras que en español general este tiempo verbal se asocia con rasgos aspectuales, de tiempo deíctico y de tiempo anafórico, en el español de contacto se vincula solamente con un rasgo modal ([evidencia indirecta]) y un rasgo de tiempo deíctico ([pasado]).

A diferencia de lo que hemos propuesto para el contacto con el guaraní, en este caso la forma verbal no se comporta como una unidad, de manera fosilizada, sino que es productiva sobre distintas raíces verbales. De este modo, no se corresponde con un único ítem de vocabulario sino con un conjunto de ellos. Así, proponemos que la mor-

\footnotetext{
${ }^{20}$ Sánchez (2004) analiza este uso del pretérito pluscuamperfecto como un caso de convergencia funcional: las formas del quechua y del español coinciden parcialmente en los rasgos que expresan, lo cual facilita la transferencia del rasgo evidencial al español.

${ }^{21}$ La selección del pret. pluscuamperfecto puede deberse a que este reproduce, en el discurso indirecto del español general, los verbos en pretérito perfecto simple (cfr. Vino $\rightarrow$ Dijo que había venido). Si se considera que los verbos analizados aquí están enmarcados por el verbo decir con valor reportativo, no resulta completamente azarosa su selección.
} 
fología flexiva presente en el auxiliar había codifica el rasgo [evidencia indirecta] mientras que el participio codifica un rasgo de [pasado]. El hecho de que el participio sea el que aporta este valor es paralelo a lo que ocurre con el pretérito perfecto compuesto en esta zona, que se comporta como el pretérito perfecto simple del resto del país (cfr. nota 15). De este modo, los auxiliares había y ha se oponen aquí en términos de evidencialidad, mientras que el participio es el que codifica el valor de pasado deíctico ${ }^{22}$.

A partir de este análisis resulta interesante resaltar que los ítemes de vocabulario correspondientes al pretérito pluscuamperfecto se alejan de las características morfológicas del morfema -sqa paralelo en la lengua indígena. En quechua, el rasgo de tiempo pasado y el de evidencialidad indirecta sufren una operación de fusión a partir de la cual ambos se codifican bajo un mismo sufijo, es decir, mediante un único ítem de vocabulario. En el español de contacto, por el contrario, la fusión no se aplica y los rasgos involucrados se reorganizan en ítemes de vocabulario separados, lo que da como resultado una forma perifrástica. Esto constituye evidencia a favor de la propuesta de Sánchez (2004) de que la transferencia entre lenguas ocurre fundamentalmente en el nivel de los rasgos abstractos, es decir, es independiente de las formas superficiales que los manifiestan en cada una de las lenguas de contacto.

\section{Los fenómenos de contacto}

El análisis de los datos nos ha permitido concluir que el contacto provoca reorganizaciones en al menos dos niveles fundamentales de una gramática: el de los rasgos sintáctico-semánticos y el morfológico. Así, dos formas a primera vista equivalentes, como son el pretérito pluscuamperfecto en ambas variedades de contacto, presentan diferencias respecto del español general que pueden ubicarse en los dos niveles analizados.

En relación con el nivel de los rasgos sintáctico-semánticos, se incorporan en el español de contacto nuevos rasgos ([percepción demorada] y [evidencia indirecta]) en cada una de las variedades. Dichas incorporaciones generan una reorganización de todo el sistema, que se hace evidente, por ejemplo, en el hecho de que en el contacto con el

${ }^{22}$ Nótese al respecto que en el pretérito perfecto compuesto, al igual que sucede con el pretérito pluscuamperfecto, se neutraliza también la oposición aspectual, pudiendo utilizarse incluso en contextos imperfectivos:

(ii) a. Diz que ahí, cuando ya ha estado carniando ['estaba carniando'] y sacando las achuras, que le dice (...). [V:276, I]

b. En eso que ha estado ['estaba'] ahí el zorro, que se han juntado muchas moscas, y diz que el zorro despacito los ha ido echando en la vejiga. [V:276, I] 
quechua la aparición del rasgo [evidencia indirecta] supone una oposición modal del pretérito pluscuamperfecto respecto del pretérito perfecto compuesto. En este sentido, no es solo la selección de un nuevo rasgo lo que está en juego aquí sino la gramaticalización de todo un sistema evidencial que establece nuevas oposiciones entre las formas existentes.

En consecuencia, podemos concluir que la presencia de la lengua indígena puede modificar el inventario de rasgos que operan en el español. En los ejemplos analizados el contacto supone la ampliación de dicho inventario. Sin embargo, esto no siempre es así sino que es posible, también, que este se vea reducido. Esto es frecuente, por ejemplo, en variedades del español en contacto con lenguas que no seleccionan género (como ocurre en las dos variedades de contacto analizadas aquí, véase nota al pie 4) o que no codifican rasgos temporales (como sucede en el contacto con el toba (qom) en el nordeste de la Argentina (cfr. Avellana, 2012). En suma, lo que en una lengua se manifiesta de manera léxica puede pasar a codificarse de manera gramatical a causa de la incorporación de un rasgo que no poseía previamente, o viceversa.

A su vez, las incorporaciones en el nivel de los rasgos sintáctico-semánticos repercuten en la morfología. En particular, observamos que en ambas situaciones de contacto el pretérito pluscuamperfecto - ya existente en el español general - se resignifica para dar cuenta de los nuevos rasgos. La resignificación de una forma existente es solo una de las tantas posibilidades que habilita el contacto de lenguas, entre las cuales se encuentran también la incorporación directa de un préstamo de la lengua indígena (como sucede con ra'e; cfr. 15), la retención de una forma propia de otro estadio del español (como ocurre con diz que; cfr. 23) o la mayor frecuencia de uso de una forma propia del español general, con igual significado.

Asimismo, las peculiaridades morfológicas que posee el pretérito pluscuamperfecto en ambas situaciones de contacto encuentran cierto correlato en las propiedades de las lenguas indígenas: mientras que en guaraní el morfema ra'e se comporta como un morfema no ligado, que codifica un único rasgo y se ubica normalmente pospuesto a algún constituyente de la oración, el morfema quechua -sqa es un sufijo que expresa más de un rasgo y que se encuentra unido morfológicamente a las raíces verbales. Esto explica la libertad posicional de había sido (que) en el contacto con el guaraní, un morfema 
libre que señala un único rasgo ${ }^{23}$, frente al uso flexivo de la morfología del pret. pluscuamperfecto en zona de contacto con el quechua.

De esta manera, en el nivel morfológico existen, por un lado, reorganizaciones sobre los ítemes de vocabulario que se producen como una consecuencia derivada de lo que ocurre en el nivel sintáctico-semántico (i.e. los rasgos abstractos que se incorporan deben recibir una expresión fonológica y los ítemes de vocabulario se resignifican para dar cuenta de ellos). Por otro lado, con menor frecuencia, también se incorporan de manera directa propiedades puramente morfológicas de la lengua indígena (como, por ejemplo, se calca la ubicación de los morfemas de la lengua indígena). Más allá de estas similitudes, creemos junto con Sánchez (2004) que la existencia de diferencias morfológicas superficiales entre las lenguas en contacto no es un impedimento para la transferencia de rasgos. Más bien el camino es el inverso: la equivalencia se establece en primera instancia en el nivel de los rasgos sintáctico-semánticos y lo que ocurre en el componente morfológico es dependiente de dicha equivalencia.

\section{Conclusiones}

Con este trabajo esperamos haber contribuido, por un lado, con el estudio del español americano en contacto con lenguas indígenas, a partir de la descripción y delimitación de un conjunto de estructuras gramaticales.

Por otro lado, la comparación de dichas estructuras con las gramáticas de las lenguas indígenas con las que están en contacto nos ha permitido corroborar ciertos procesos de transferencia. En este sentido, hemos justificado mediante herramientas gramaticales la existencia de dichos procesos y, al mismo tiempo, hemos profundizado en el conocimiento de las estructuras de contacto por medio del trabajo contrastivo. A partir de esto, podemos concluir que el pretérito pluscuamperfecto en ambas variedades adopta características particulares, transferidas de las lenguas indígenas respectivas.

Asimismo, hemos ahondado en el análisis teórico de los procesos de transferencia identificados y hemos concluido que estos ocurren tanto en el nivel de los rasgos abstractos como en las características propias del componente morfológico. En este sentido, corroboramos que el contacto de lenguas puede llevar a la modificación del inventario de rasgos activos de cada lengua y que los fenómenos de transferencia no son me-

\footnotetext{
${ }^{23}$ No obstante, es necesario remarcar que la tendencia a que las transferencias se manifiesten mediante morfemas libres es algo frecuente en las situaciones de contacto y no siempre guarda relación con las propiedades morfológicas (aislantes, aglutinantes o fusionales) de las lenguas involucradas.
} 
ramente superficiales sino que pueden transformar aspectos sustanciales de la gramática de una lengua. De igual forma, determinamos que las propiedades transferidas en cada uno de los dos niveles considerados son independientes y pueden (y deben) ser analizadas de ese modo.

Finalmente, hemos argumentado que los aspectos sujetos a transferencia coinciden, precisamente, con aquellos lugares en los que, desde la propuesta de la Morfología Distribuida, puede sistematizarse la variación interlingüística. Por esto, consideramos que el estudio del contacto de lenguas aporta un conjunto de datos relevantes para la discusión sobre los aspectos universales y particulares de las lenguas, dado que todas aquellas propiedades que pueden ser transferidas son, a su vez, las que están sujetas a variación.

En líneas generales, esperamos haber provisto un análisis gramatical detallado que confirma que los hechos de transferencia no son fenómenos aislados ni caóticos. Por el contrario, dichos fenómenos se vinculan con propiedades gramaticales concretas, que pueden formalizarse dentro de un sistema en el cual una modificación en un aspecto de la gramática puede conllevar cambios en otros aspectos del sistema. Asimismo, la coexistencia con otra lengua no genera solo simplificaciones sobre la gramática de contacto sino que, por el contrario, provoca complejas interrelaciones entre lo que se expresa por medio de elementos gramaticales o léxicos en cada lengua. En suma, las propiedades de una lengua pueden incorporarse en otra, generando una nueva gramática con características que resultan innovadoras para el español general pero que son tan gramaticales como las de cualquier otra lengua natural.

Recibido: 22-VII-2012

Aceptado: 20-II-2013

\section{Referencias bibliográficas}

Adelaar, Willem (1977): Tarma Quechua: Grammar, Texts, Dictionary. Lisse, Peter de Ridder Press.

Aikhenvald, Alexandra (2003): "Mechanisms of Change in Areal Diffusion: New Morphology and Language Contact”, Linguistics, 39, págs. 1-29.

Aikhenvald, Alexandra (2004): Evidentiality. Oxford, Oxford University Press.

Albarracín, Lelia, Mario Tebes y Jorge Alderetes (2002): Introducción al Quichua Santiagueño por Ricardo Nardi. Buenos Aires, Dunken.

Angulo, Stella Maris, Emilene Núñez Campos y Andrea Chaves (2011): "República del Paraguay" (ms.). Disponible en: http://www.slideshare.net/hadita10/paraguay9257927.

Avellana, Alicia (2012): Las categorías funcionales en el español en contacto con lenguas 
indígenas en la Argentina: tiempo, aspecto y modo. Tesis de doctorado, Universidad de Buenos Aires.

Cerrón Palomino, Rodolfo (1994): Quechumara; Estructuras paralelas de las lenguas Quechua y Aimara. La Paz, CIC.

Cerrón Palomino, Rodolfo (2003): Castellano andino. Aspectos sociolingüísticos, pedagógicos y gramaticales. Lima, Pontificia Universidad Católica del Perú.

Cinque, Guglielmo (1999): Adverbs and functional heads: A cross-linguistic perspective. Oxford, Oxford University Press.

Chomsky, Noam (1981): Lectures on Government and Binding. Dordrecht, Foris.

Chomsky, Noam (1986): Knowledge of Language, its Nature, Origin and Use. New York, Praeger.

Chomsky, Noam (1993): “A Minimalist Program for Linguistic Theory”. En Hale, K. y S. Keyser (eds.): The View from Building 20: Essays in Linguistics in Honor of Sylvain Bromberger. Cambridge, MIT Press, págs. 1-52.

Chomsky, Noam (1995): El Programa Minimalista. Madrid, Alianza.

Cusihuamán, Antonio (1976): Gramática quechua. Cuzco-Collao. Lima, Instituto de Estudios Peruanos.

DeLancey, S. (1997) "Mirativiy: The grammatical marking of unexpected information". Linguistic Typology, 1, págs. 33-52.

Dietrich, Wolf (2010): “Tiempo, aspecto y evidencialidad en guaraní”, Liames, 10, págs. 67-83.

Faller, Martina (2004): "The Deictic Core of 'Non-Experienced Past' in Cuzco Quechua", Journal of Semantics, 21, págs. 45-85.

Faller, Martina (2007): "The Cusco Quechua Reportative Evidential and Rhetorical Relations" (ms.). Disponible en: http://personalpages.manchester.ac.uk/staff/ martina.t.faller.

Giorgi, Alessandra y Fabio Pianesi (1997): Tense and Aspect: From Semantics to Morphosyntax. Oxford, Oxford University Press.

González Torres, Dionisio (1992): Cómo nos expresamos particularmente en nuestro español y en jopará. Disponible en: http://www.portalguarani.com/obras_autores_ detalles.php?id_obras=14916.

Granda, Germán de (1998): "De nuevo sobre quechua y español en el noroeste argentino. Reexamen de algunos temas". Lexis, XXII, 1, págs. 1-10.

Halle, Morris y Alec Marantz (1993): "Distributed Morphology and the Pieces of Inflection”. En Hale, K. y S. Keyser (eds.): The View from Building 20. Cambridge, MIT Press, págs. 111-176.

Halle, Morris y Alec Marantz (1994): “Some Key Features of Distributed Morphology”. 
En Carnie, A; H. Harley y T. Bures (eds.): Papers on Phonology and Morphology. MITWPL, 21, págs. 275-288.

Hardman de Bautista, Martha (1982): "The mutual influence of Spanish and the Andean languages”, Word, 33, 1-2, págs. 143-157.

[Ka] Kany, Charles (1969): Sintaxis hispanoamericana. Madrid, Gredos.

Klee, Carol y Alicia Ocampo (1995): "The expression of Past Reference in Spanish Narratives of Spanish-Quechua Bilingual Speakers". En Silva Corvalán, C. (ed.): Spanish in Four Continents. Studies in language contact and bilingualism. Washington, Georgetown University Press.

Kornfeld, Laura (2004): Formación de palabras en la sintaxis desde la perspectiva de la Morfología Distribuida.Tesis de doctorado, Universidad de Buenos Aires.

Krivoshein, Natalia y Feliciano Acosta (2007): Gramática guaraní. Asunción, Servilibro.

Lefebvre, Claire y Pieter Muysken (1988): Mixed categories: Nominalizations in Quechua. Dordrecht, Kluwer Academic Publishers.

Liuzzi, Silvio y Pablo Kirtchuk (2010): “Tiempo y aspecto en guaraní”, Amerindia, 14, págs. 9-41.

Martín, Herminia (1976): “Un caso de interferencia en el español paceño", Filología, XVII-XVIII, págs. 119-130.

Melià, Bartomeu, Luis Farré y Alfonso Pérez (1997): El guaraní a su alcance. Un método para aprender la lengua guaraní del Paraguay. Asunción, CEPAG.

Merma Molina, Gladys (2007): Contacto lingüístico entre el español y el quechua: un enfoque cognitivo-pragmático de las transferencias morfosintácticas en el español andino peruano. Tesis de doctorado, Universidad de Alicante.

Olbertz, Hella (2005): "Dizque en el español andino ecuatoriano: conservador e innovador”. En Olbertz, H. y P. Muysken (eds.): Encuentros y conflictos: bilingüismo y contacto de lenguas. Madrid/Frankfurt, Iberamericana/Vervuert, págs. 77-94.

Olbertz, Hella (2009): "Mirativity and exclamatives in functional discourse grammar: evidence from Spanish" En Keizer, E. y G. Wanders (eds.): The London papers I. Web Papers in Functional Discourse Grammar, vol. 82. Amsterdam, Functional Grammar Foundation, págs. 66-82.

Palacios Alcaine, Azucena (1999): Introducción a la lengua y cultura guaraníes.Valencia, Universitat de València.

Palacios Alcaine, Azucena (2008): "Paraguay". En Palacios Alcaine, A. (coord.): El español en América. Barcelona, Ariel, págs. 279-300.

Reuse, Willem de (2003): "Evidentiality in Western Apache (Athabascan)". En Aikhenvald, A. y R. M. W. Dixon (eds.): Studies in Evidentiality. Amsterdam/Philadelphia, John Benjamins, págs. 79-100.

Sánchez, L. (2004): "Functional convergence in the tense, evidentiality and aspectual 
systems of Quechua Spanish bilinguals", Bilingualism: Language and Cognition, 7, 2, págs. 147-162.

Schumacher de Peña, Gertrude (1975): "Observaciones sobre el sistema verbal del español andino". En Materiales descriptivos para la enseñanza del castellano como segunda lengua. Lima, Instituto Nacional de Investigación y Desarrollo de la Educación, págs. 63-104.

Velázquez-Castillo, Maura (2010): Handout de la comunicación "Alineamiento semántico y el sistema Aspecto-Modal (AM): El caso del guaraní", presentada en el $I V$ Simposio sobre el estado actual del conocimiento del Gran Chaco Meridional (XXX Encuentro de Geohistoria Regional), Resistencia, 19-21 de agosto.

Vendler, Zeno (1967): Linguistics in Philosophy. Ithaca and London, Cornell University Press.

Vidal de Battini, Berta (1980): Cuentos y leyendas populares de la Argentina. Buenos Aires, Ediciones culturales argentinas.

Páginas web (consultadas entre octubre de 2011 y junio de 2012):

[W1] http://www.foro.fullaventura.com

[W2] http://www.lajaula.com.py

[W3] http://twitoaster.com

[W4] http://www.elforro.com

[W5] http://www.diariopopular.com.py

[W6] http://twitter.com

[W7] http://www.tocorre.com

[W8] http://www.taringa.net

[W9] http://www.diarioparaguayo.com

[W10] http://www.lanpy.com

[W11] http://tvparaguayadesplieguecomentarios.blogspot.com

[W12] http://www.franjanegra.com

[W13] http://www.franjeado.com

[W14] http:// motores.com.py

[W15] http://clubcerro.mforos.com

[W16] http://www.contrapuntoya.com.ar

[W17] http://twitoaster.com

[W18] http://www.popular.com.py

[W19] http://www.portalguarani.com

[W20] http://www.cronica.com.py 